After the case has terminated the building occupied by the sick person, and the contiguous buildings, should be fumigated witl some good insecticide. I prefer sulphur wherever possible. Where sulphur can not be used I recommend pyrethrum. It is very important that the man at the head of affairs should have a thorough knowledge of the details of all these matters. He should know whether the screening is properly done; when fumigating, whether the pasting has made the building sufficiently tight, etc. It requires considerable experience to become expert in all these details, and a mere theoretical knowledge is at first not of much avail.

For the small community, above described, these measures of caring for the sick and destroying infected mosquitoes need not add to the force. The health officer himself would have plenty of time to look after the sick. 'The chief carpenter with his squad could do the screening, and the inspector in charge of the cleaning squad could do the fumigating.

The relative cost would, of course, decrease as the size of the municipality increased. Take a municipality ten times the size of the one described, with a population of about 30,000 people, who would live in some 6,000 louses. The personnel would be increased about as follows:

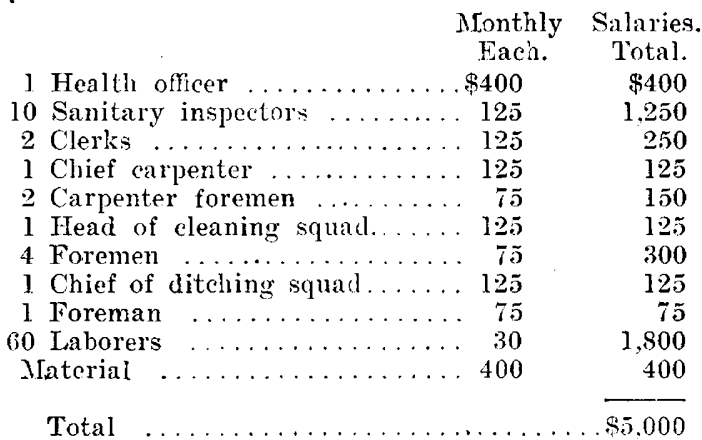

Here you see that our unit of 600 houses, instead of costing $\$ 1,900$ per month, would cost only $\$ 500$ per month, and, of course, as the municipality increases in size the cost per unit would continue to decrease. In this estimate for a large municipality $I$ am not includ. ing the cost of care of the sick, as the municipality of this size would necessarily have some hospital in which the sick would be cared for.

In the municipality the same measures that are directed against yellow fever will avail against malaria. This has occurred in Havana, Cuba. The anti-mosquito work done there in the past ten years has practically eliminated malarial ferer. The deaths from malaria prior to 1896 areraged about 250 per year. This has gradually failen until in the last year or two they have had less than fifty deaths per year. In a city sitnated as is Havana, this, I think, means the extinction of malaria. The fifty deaths occurring every year at present in Havana, due to malaria, are probably caused by the persons affected by malaria who come in from the rest of the island. Havana is the metropolis of Cuba; all the large hospitals are located there, and a great many people, whenever taken sick, come in from all parts of the island to these hospitals for treatment. In the ordinary course of affairs a large number of malaria patients come into the city from the rest of the island in connection with their routine business.

Great strides in diminishing the spread of yellow fever all over the world have been made in the last ten years. Ten years ago the four principal centers of yel- low fever in the world were Vera Cruz, Havana. Rio Taneiro and Guaraquil. Of these Havana and Rio $\mathrm{Ja}$ neiro were much more important centers of infection than Vera Cruz and Guayaquil. From Havana yellow lever has practically been eliminated, and within the last six months Rio Janeiro has been almost as suecessful. In Vera Cruz yellow fever has become sporadic instead of being endemic. Sporadic cases within the last rear have occurred in several different parts of tropical America, and a few on the west coast of Africa, but nothing comparable with what it was ten years ago. It seems to me that vellow fever will entirely disappor within this generation, and that the next generation will look on yellow fever as an extinct disease having only a historic interest. They will look on the yellow fever parasites as we do on the three-toed horse-as an animal that existed in the past, without any possibility of reappearing on the earth at any future time.

Concerted effort on the part of the countries in which it now occurs, I think, would extinguish the disease at once at no very great expense and without undue labor. I believe that if this congress could get the governments of the various countries in which yellow fever has occurred during the past rear to agree to keep such an organization as I have outlined, which could be sent at once to the locality where yellow fever appears, and there stamp it out, at the end of two years yellow fever would have disappeared from the western hemisphere.

\section{THE FUNCTION OF THE END-ORGANS IN THE VESTIBULE AND SEMICIR- CUIAAR CANALS}

AND SOME METHODS FOR EXAMINING THESE CANALS IN PRACTICAL DIAGNOSIS

GEORGE E. SHAMBAUGH, M.D.

CHICAGO

In order to understand the disturbances in function arising from the diseases of the vestibule and"semicircular canals, as well as the principles involved in diagnosing these conditions, it is necessary that one keep clearly in mind a few fundamental facts in regard to the anatomy and the physiology of these structures.

Only a few years ago physiologists looked on the internal ear as a mechanism that had to do solely with the function of hearing. This function they assigned to the three parts of the labyrinth as follows: To the end-organs in the cochlea was attributed the function of tone perception; to the end-organs in the vestibule the function of noise perception, and to the semicircular canals was attributed the function of determining the direction from which sound comes.

The more recent studies of the internal ear justify the conclusion that the cochlea alone is interested in sound perception, whether this be in the nature of noises or musical tones, and that the end-organs in the vestibule and semicircular canals have to do with the important function of preserving the equilibrium. This latter function is accomplished by these several endorgans as follows: From the vestibule we get our knowledge of position in space; from the semicircular canals our knowledge of turning motions. The data derived from these two sets of end-organs seems to be sufficient to account for the function of equilibrium.

The function attributed to the vestibule is accomplished as follows: There are located in the vestibule two end-organs, identical in structure, but placed on 
planes at right angles to each other. These are the socalled macula acustica of the utricle and the macula acustica of the saccule. These end-organs consist of a large number of hair-bearing cells. The hairs from these cells project into a superimposed jelly-like structure, in which there is imbedded a large number of small crystals of oxalate of lime, called otoliths. The otoliths give to this otolith membrane a specific gravity slightly greater than the surrounding endolymph. These end-organs in the vestibule perform their function of giving us our idea of position in space as follows: Placed at right angles as they are to each other, with each change in the position of the head, the otolith membranes, owing to their greater specific gravity, either press or drag on the hairs of the underlying hair cells in such a way as to produce a different kind of stimulus for each different position assumed.

The semicircular canals are three in number and occupy approximately the three planes in space. Each canal is provided with an expansion at one end, called the ampulla. In this ampulla is located the end-organ for the canal, which is constructed as follows: A ridge from the outer wall projects into the space of the am-

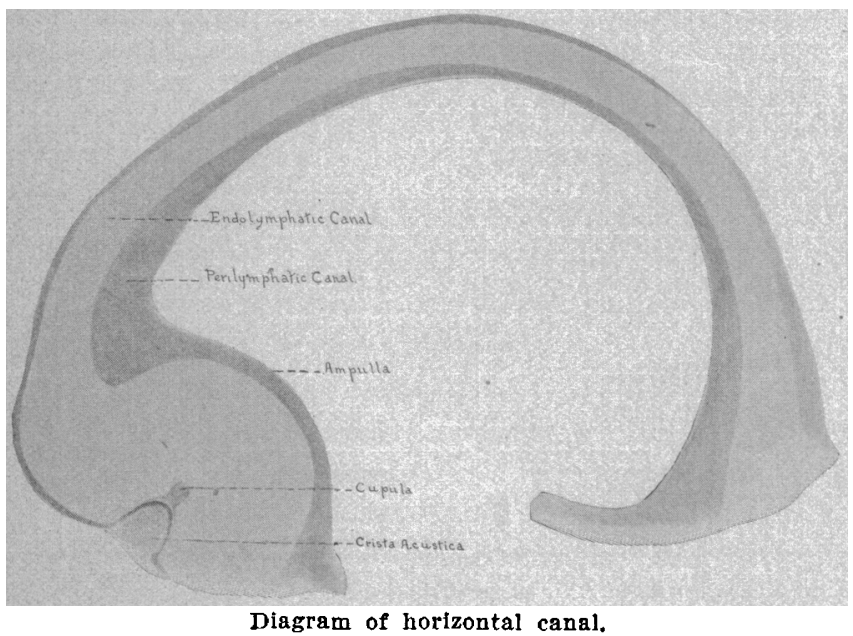

pulla and is covered with the hair-bearing neuroepithelium. This ridge is known as the crista acustica ampullaris. Capping the ridge is a peculiar elastic structure, called the cupula, with the under surface of which the hairs of the hair cells come in direct contact. This entire apparatus is so arranged that any motion in the endolymph of the semicircular canal must impinge directly against this cupula and tends to bend it over in one direction or the other. A turning motion of the head in any plane whatsoever must necessarily result in a motion of the endolymph in one or several of these semicircular canals, thus producing a stimulation of the hair cells in their respective ampullæ.

In order to get a clear idea of the physiology of the semicircular canals we have but to attach to the inside of a bucket filled with water a tuft of elastic hairs. Let this tuft represent the cupula placed over the crista acustica of the ampulla, then a bending over of this tuft in either direction would constitute a stimulation of the underlying hair cells of the crista ampullaris. If now the bucket is rotated very slowly the water will be able to take on the motion of the sides of the bucket, and the tuft os hair will not be bent over, and there will result no stimulation of the hair cells. When the bucket is rotated more rapidly the tuft will be bent backward in a direction opposite to the motion of the bucket, being carried back by the water which does not, for some time, take on the motion of the turning bucket. If, however, the turning of the bucket is kept up long enough the water will finally be turning just as rapidly as the bucket itself, and again the tuft of hairs will project straight out into the water and will not be bent to either side. If the bucket is suddenly stopped the water will continue whirling for some time, and the tuft will now be bent over again, but this time in the same direction as that in which the bucket was being turned.

After witnessing this demonstration with the turning bucket of water one ean readily understand the following phenomena that present themselves when a person is placed on a rotating chair: If the chair is rotated very slowly no sense of turning. is experienced, provided the eyes are closed, for the reason that the endolymph in the semicircular canals takes on the motion of the walls of the canal and the cupula is bent neither this way nor that. If the turning is more rapid a sense of turning is readily experienced, at the same time a reflex eye symptom will become apparent. This is a nystagmus, increased when the eyes are turned in the direction of the rotation. In case the whirling continues long enough the person will lose all sense of turning and the nystagmus will cease, for the reason that the endolymph in the semicircular canals takes on the motion of these canals and the cupula is not pressed on, and so can not stimulate the hair cells. On stopping suddenly the individual will immediately experience vertigo and the nystagmus will return, but now it will be directed to the side opposite to the turning, just as would be expected from an observation of the experiment with the bucket of water. With the rotating chair it is possible to demonstrate that there is destruction of the semicircular canal apparatus such as occurs in epidemic cerebrospinal meningitis. In these cases neither vertigo nor nystagmus can be produced by turning, and such persons do not experience seasickness.

Besides this experiment of the rotating chair there are several other methods of examining the semicircular canals; two of these methods are dependent on the production of a motion in the endolymph in the canals.

In 1892 Ewald performed the interesting experiment of making a small opening in the horizontal canal of the pigeon, into which he fixed a small canula, and found that when pressure was exerted on the fluid in the canal by compressing a rubber bulb attached to the canula, marked nystagmus was readily induced. This experiment is successfully used in diagnosing the presence of an opening into the labyrinth such as sometimes occurs by ezosion in cases of cholesteatoma. All that is required in these cases to produce vertigo and nystagmus is to compress or rarefy the air in the external meatus.

The third method of examining the condition of the semicircular canals is that recently elaborated by Barany of Vienna. This consists of the production of a motion in the endolymph of the semicircular canals by chilling or warming the part of the capsule of the labyrinth exposed in the tympanum and antrum by syringing the external canal with cold or with warm water. The motion in the endolymph produced by syringing the ear with cold water will, of course, be just opposite to that produced by syringing with warm water. Using water at the temperature of the body will naturally produce no motion in the endolymph. The stimulation of the semicircular canals by means of hot or cold water 
will be much more readily obtained in cases in which a large perforation of the membrana tympani permits the water to come into direct contact with the wall of the labyrinth. This stimulation is also the more readily obtained by using water quite warm or quite cold. The force of the stream has naturally no influence on this stimulation.

The study of the occurrence of vertigo and nystagmus, both that observed in disease of the labyrinth ard that brought on by syringing the ear with hot and cold water, has established the following facts, which are of great assistance in diagnosis not only of the labyrinth but of the cerebellum as well:

1. If the semicircular canals are normal and the ear is syringed with cold water, vertigo will result, and there will be set up a nystagmus increased by directing the eyes toward the opposite side. If the ear is syringed with warm water the same symptoms will occur, but the nystagmus will be toward the same side.

2. Should there exist an irritation of the endings of the vestibular nerve in the labyrinth, such as may be occasioned by a circumscribed suppuration in the labyrinth; there will be spontaneous nystagmus directed toward the same side. Syringing the ear with cold water will produce a positive reaction.

3. If there occurs a sudden destruction of endings of the vestibular nerve, such as would be occasioned by a diffuse suppuration in the labyrinth, there will be set up a spontaneous nystagmus directed toward the opposite side, but lasting only from a few days to several weeks. This nystagmus has its origin in the opposite normal ear. Syringing the affected ear with hot and cold water produces no response.

4. In case of long standing destruction of the nerve endings in the vestibular nerve, such as occurs in chronic diffuse labyrinth suppuration, there will be no spontaneous nystagmus and no reaction can be obtained by syringing the ear with hot or cold water.

5. In case of cerebellár disease, such as cerebellar tumor, cerebellar abscess or a meningitis in this locality, there will occur a spontaneous nystagmus directed toward the affected side.

It follows, therefore, that in case there exists pronounced rotating nystagmus, and the membrana tympani is normal, showing an absence of an inflammation in the middle ear, the presence of a cerebellar tumor should be suspected. On the other hand, should there exist pronounced. spontaneous rotating nystagmus in a case of suppurative otitis media without ferer, but with severe deafness," and this nystagmus is directed toward the affected side, , while, the syringing of the ear with cold water produces no response, the diagnosis of a cerebellar abscess is the probable one. Here the complete destruction of the hearing in the affected ear would indicate a probable diffuse suppuration of the labyrinth. The failure to get the caloric response on syringing points to destruction of end-organs in the semicircular canals. The only spontaneous nystagmus which this lesion could produce would be directed toward the opposite side. The absence of rise of temperature would in most cases eliminate a meningitis as the source of the nystagmus, while a cerebellar abscess pressing on the vestibular nerve could produce a spontaneous rotating nystagmus, which would be increased by directing the eyes toward the affected side.

100 State Street.

\section{INTESTINAL LOCALIZATION}

WITH CERTAIN OTHER SUBJECTS RELATING TO THE SURGERY AND SURGICAL ANATOMY OF THE SMALL INTESTINE AND ITS MESENTERY

\section{GEORGE H. MONKS, M.D.}

Surgeon to the Boston City Hospital; Lecturer In Surgery at the Harvard Medical School BOSTON

This article is, with a few changes, the text of a demonstration on the cadaver which I gave in Boston, May 1, 1908, by invitation, before the Society of Clinical Surgery. The subjects considered have little to connect them, except that all of them relate to the surgery, or to the surgical anatomy, of the small intestine and its mesentery. The procedures described are taken mostly from cadaver studies which I have made from time to time. Some of these studies have already been published at greater length in former articles. ${ }^{1}$ The object of the present article is to present, in a form as condensed and practical as possible, some of the procedures with which these studies were concerned. An effort has been made to elucidate the text by a free use of illustrations-a method which, useful as it may be in many instances, is unfortunately not wholly satisfactory as a substitute for actual demonstration.

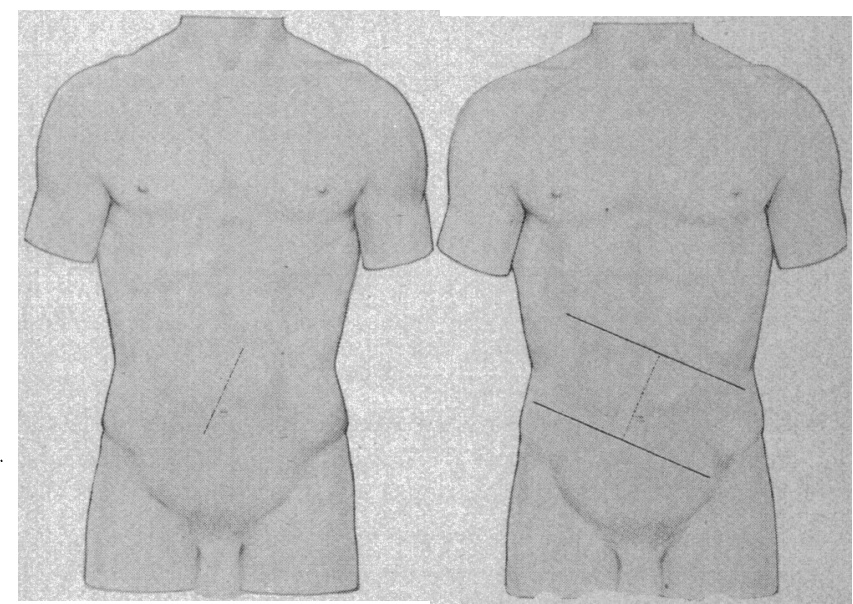

Fig. 1.-Diagram showing on the abdominal wall a surface the abdominal wall a surface proximately with the root of the mesenterg. (This diagram and
the next one are taken from a the next one are taken from a figure has been used.)

While some of the procedures described have repeat. edly been employed with success in operations, otherswhich have not been used in operations-are presented merely as procedures which, from an anatomic point of: view, are possible. Others, again, are simply anatomic. demonstrations.

\section{THE MESENTERY}

As the mesentery will frequently be referred to in this article, it is desirable at the outset to make a few general remarks about this structure, especially in rogard to its root, its shape, and the disposition of its different parts.

The Root of the Mesentery.-The part of the mesentery where the vessels enter is, as pointed out by 'Treves,

1. Intestinal Localization, Ann. Surg., October, 1903; Studies in the Surgical Anatomy of the Small Intestine and its Mesentery, Ann. Surg., October, 1905 ; Experiments in Flushing the Intestina; Canal with Salt Solution Through Multiple Enterotomy Openfings, Canal with Salt Solution 